\title{
Nonlinear Propagation of Leaky TE-Polarized Electromagnetic Waves in a Metamaterial Goubau Line
}

\author{
Eugene Smolkin and Yury Smirnov \\ Penza State University \\ Department of Mathematics and Supercomputing, Penza State University, \\ Krasnaya Str. 40, 440026 Penza, Russia \\ E-mail(corresp.): e.g.smolkin@hotmail.com \\ E-mail: smirnovyug@mail.ru
}

Received June 30, 2020; revised April 23, 2021; accepted April 24, 2021

\begin{abstract}
Propagation of leaky TE-polarized electromagnetic waves in the Goubau line (a perfectly conducting cylinder covered by a concentric dielectric layer) filled with nonlinear metamaterial medium is studied. The problem is reduced to the analysis of a nonlinear integral equation with a kernel in the form of the Green function of an auxiliary boundary value problem on an interval. The existence of propagating nonlinear leaky TE waves for the chosen nonlinearity (Kerr law) is proved using the method of contraction. For the numerical solution, a method based on solving an auxiliary Cauchy problem (a version of the shooting method) is proposed. New propagation regimes are discovered.
\end{abstract}

Keywords: leaky TE waves, metamaterial Goubau Line, nonlinear permittivity, nonlinear eigenvalue problem, numerical method.

AMS Subject Classification: 78A60; 34B15; 34L16.

\section{Introduction}

For many years, the theory of the polarized electromagnetic wave propagation in planar and cylindrical waveguides in the linear case has been developing $[2,26,28]$. This theory is important because, firstly, such problems describe real physical processes that are important both for applied and theoretical sciences and, secondly, from the mathematical point of view, this theory is an affluent source of sophisticated and interesting mathematical problems.

Copyright (C) 2021 The Author(s). Published by Vilnius Gediminas Technical University This is an Open Access article distributed under the terms of the Creative Commons Attribution License (http://creativecommons.org/licenses/by/4.0/), which permits unrestricted use, distribution, and reproduction in any medium, provided the original author and source are credited. 
The problem of the wave propagation in open waveguides constitutes an important class of vector electromagnetic problems. A perfectly conducting cylinder covered by a concentric dielectric layer, the Goubau line (GL), is the simplest type of such guiding structures. A mathematical investigation of symmetric surface modes in a GL is performed in [4, 5, 6,27$]$. It may be important both from the theoretical standpoint and in view of further applications of GLs, to extend these results for much broader families of GLs with nonlinear metamaterial media. This will be the first necessary step to analyze more complicated open metal-dielectric waveguides with such fillings. The occurrence and analysis of leaky waves together with their various applications in microwave engineering have been a subject of numerous studies since the early 1950s (see $[8,9,10])$.

In 1967, Russian physicist V.G. Veselago predicted an extraordinary electromagnetic wave phenomenon which is related to materials with a simultaneously negative permittivity and negative permeability [29]. He hypothetically created a lossless meta-material and showed the extraordinary properties of this material which is not found in nature, in particular, negative group velocity, negative refraction, the reversal of the Doppler effect and Cherenkov radiation.

The present study focuses on the analysis of leaky electromagnetic wave propagation in a Goubau Line filled with a nonlinear metamaterial medium. Metamaterial is an artificial material with negative permittivity and negative permeability (see $[12,29,30]$ ). The nonlinearity is expressed by the Kerr law $[3,7,13]$. The main task which we resolve is to elaborate mathematically correct problem statements for nonlinear differential equations that enable one to introduce and investigate eigenmodes in an open nonlinear guiding medium, calculate propagation constants, and reveal fundamental properties of eigenmodes. The propagation constants will be determined as roots of the commonly used dispersion equations.

This framework has not been addressed in the literature, to the best of our knowledge. A background study concerning nonlinear guided waves in media with Kerr and Kerr-like nonlinearities can be found in $[14,15,20,24]$. In papers $[16,17,18,19,21,22]$ numerical study in layered nonlinear dielectric and metal-dielectric waveguides is performed. Numerical results are obtained with the help of a version of the shooting method (see e.g. [16, 23, 25]).

\section{Statement of the problem}

Let us consider three-dimensional space $\mathbb{R}^{3}$ with a cylindrical coordinate system $O \rho \varphi z$. The space is filled with an isotropic and nonmagnetic medium (it is supposed that everywhere $\mu=\mu_{0}$, where $\mu_{0}$ is the permeability of free space) having the constant permittivity $\varepsilon_{0}=$ const where $\varepsilon_{0}$ is the permittivity of free space, without sources. A perfectly conducting cylinder covered by a dielectric layer with a cross-section

$$
\Sigma:=\left\{(\rho, \varphi): r_{0} \leqslant \rho \leqslant r, 0 \leqslant \varphi<2 \pi\right\}
$$

and a generating line parallel to the axis $O z$ is placed in $\mathbb{R}^{3}$. 
The cross section of GL consists of two concentric circles of radii $r_{0}$ and $r$ (see Figure 1): $r_{0}$ is the radius of the internal (perfectly conducting) cylinder and $r_{0}-r$ is the thickness of the external (dielectric) cylindrical shell.

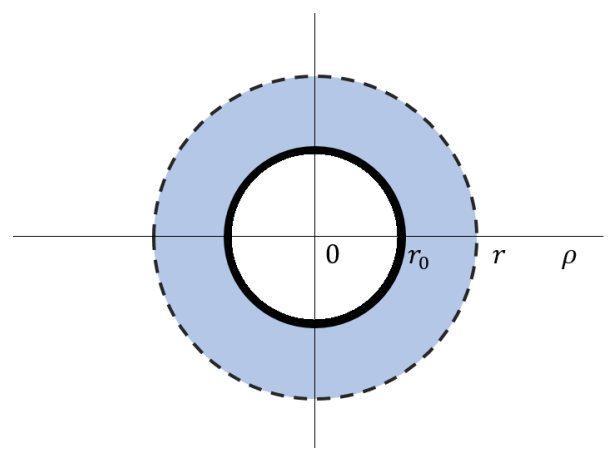

Figure 1. Goubau line; a single wire conductor coated with dielectric material.

Complex amplitudes E, $\mathbf{H}$ of the electromagnetic field satisfy Maxwell's equations [11]

$$
\left\{\begin{array}{l}
\operatorname{rot} \mathbf{H}=-i \omega \widetilde{\varepsilon} \varepsilon_{0} \mathbf{E} \\
\operatorname{rot} \mathbf{E}=i \omega \mu_{0} \widetilde{\mu} \mathbf{H}
\end{array}\right.
$$

have continuous tangential field components on the media interface $\rho=r$; and obey the radiation condition at infinity: the electromagnetic field increase as $\rho \rightarrow \infty$ in the region $\rho>r$.

We assume that permittivity and permeability in the entire space have the form $\widetilde{\varepsilon} \varepsilon_{0}$ and $\widetilde{\mu} \mu_{0}$, where

$$
\widetilde{\varepsilon}=\left\{\begin{array}{ll}
-\varepsilon+\widetilde{\alpha}|\mathbf{E}|^{2}, & r_{0} \leqslant \rho \leqslant r, \\
1, & \rho>r,
\end{array}, \widetilde{\mu}= \begin{cases}-\mu, & r_{0} \leqslant \rho \leqslant r \\
1, & \rho>r,\end{cases}\right.
$$

and $|\mathbf{E}|^{2}=\left|\left(\mathbf{E} e^{-i \omega t}, \mathbf{e}_{\rho}\right)\right|^{2}+\left|\left(\mathbf{E} e^{-i \omega t}, \mathbf{e}_{\varphi}\right)\right|^{2}+\left|\left(\mathbf{E} e^{-i \omega t}, \mathbf{e}_{z}\right)\right|^{2} ; \mathbf{e}_{k}$ is the orthonormal vector in the $k$ direction; $(\cdot, \cdot)$ is the Euclidean scalar product; and $\varepsilon, \mu$ and $\widetilde{\alpha}$ are real positive constants.

\section{TE-polarized leaky waves}

Consider TE-polarized waves in the harmonic mode (see [11]),

$$
\mathbf{E} e^{-i \omega t}=e^{-i \omega t}\left(0, E_{\varphi}, 0\right)^{T}, \mathbf{H} e^{-i \omega t}=e^{-i \omega t}\left(H_{\rho}, 0, H_{z}\right)^{T},
$$

where $\mathbf{E}, \mathbf{H}$ are complex amplitudes,

$$
E_{\varphi}=\mathrm{E}_{\varphi}(\rho) e^{i \gamma z}, \quad H_{\rho}=\mathrm{H}_{\rho}(\rho) e^{i \gamma z}, \quad H_{z}=\mathrm{H}_{z}(\rho) e^{i \gamma z}
$$

and $\gamma$ is an unknown (spectral) parameter. 
Let $k_{0}^{2}=\omega^{2} \mu_{0} \varepsilon_{0}$. Substituting components (3.1) into (2.1) and using the notation $u(\rho):=\mathrm{E}_{\varphi}(\rho)$ we obtain

$$
\left(\rho^{-1}(\rho u)^{\prime}\right)^{\prime}+\left(k_{0}^{2} \widetilde{\varepsilon \mu}-\gamma^{2}\right) u=0,
$$

where $\widetilde{\varepsilon}$ and $\widetilde{\mu}$ are defined by formula $(2.2)$.

Let us denote by $\beta^{2}:=\gamma^{2}-k_{0}^{2}$ a new spectral parameter. In the domain $\rho>r$ equation (3.2) takes the form

$$
u^{\prime \prime}+\rho^{-1} u^{\prime}-\rho^{-2} u-\beta^{2} u=0 .
$$

In the domain $r_{0} \leqslant \rho \leqslant r$

$$
u^{\prime \prime}+\rho^{-1} u^{\prime}-\rho^{-2} u+\left(\chi^{2}-\beta^{2}\right) u=\alpha u^{3},
$$

where $\alpha:=\widetilde{\alpha} \omega^{2} \mu \varepsilon_{0}, \chi^{2}:=k_{0}^{2}(\varepsilon \mu-1)>0$.

In the general case the solution of equation (3.3) for $\rho>r$ has the form

$$
u=\widetilde{C} I_{1}(\beta \rho)+C K_{1}(\beta \rho), \quad \rho>r,
$$

where $I_{1}$ and $K_{1}$ are the modified Bessel function (Infield and Macdonald function) and $\widetilde{C}, C$ are arbitrary constants.

We will consider leaky waves. More precisely, the choice of $C I_{1}(\beta \rho)$ at $\rho>r$ determines the leaky waves (see [26]) increasing at infinity because $I_{1}(\beta \rho) \rightarrow \infty$ as $\rho \rightarrow \infty$ (see [1]). In accordance with the condition at infinity solution (3.5) for leaky waves takes the form

$$
u=C I_{1}(\beta \rho), \quad \rho>r,
$$

Continuity conditions for functions $u$ and $u^{\prime}$ result from the transmission conditions for the tangential field components $\left(E_{\varphi}\right.$ and $\left.H_{z}\right)$ and have the form

$$
\left.[u]\right|_{\rho=r}=0,\left.\left[u^{\prime} / \widetilde{\mu}\right]\right|_{\rho=r}=0,
$$

where $\left.[v]\right|_{\rho=s}=\lim _{\rho \rightarrow s-0} v(\rho)-\lim _{\rho \rightarrow s+0} v(\rho)$ is the jump of the limit values of the function at a point $s$. Tangential electric field $E_{\varphi}$ vanishes at $\rho=r_{0}$ of a perfect conductor, so that

$$
u\left(r_{0}\right)=0
$$

Formulate the transmission eigenvalue problem (problem $P$ ) to which the problem of leaky waves propagating in a metamaterial rod is reduced.

Find quantities $\beta \in \mathbb{R}^{+}$such that, for a given $C \neq 0$, there is a nonzero function $u(\rho ; \beta)$ defined by formula (3.6) for $\rho>r$ which solves equation (3.4) for $r_{0}<\rho<r$ and satisfies transmission and boundary conditions (3.7) and (3.8), respectivelly. 


\section{Nonlinear integral equation}

To show the existence of eigenvalues of problem $P$ we will study solvability of the equation

$$
L u=\alpha \rho u^{3}
$$

where $L=\frac{d}{d \rho}\left(\rho \frac{d}{d \rho}\right)+\left(\kappa^{2} \rho-\rho^{-1}\right), \kappa^{2}=\chi^{2}-\beta^{2}, \rho \in\left[r_{0}, r\right]$.

Construct the Green functions $G$ for the boundary value problems:

$$
\left\{\begin{array}{l}
L G=-\delta(\rho-s), \\
\left.G\right|_{\rho=r_{0}}=\left.G\right|_{\rho=r}=0, \quad r_{0}<s<r .
\end{array}\right.
$$

It can be proved [14] that the Green functions have the forms

$$
G(\rho, s)=\frac{\pi}{2} \begin{cases}\frac{u_{l}(\rho) u_{r}(s)}{w}, & \rho<s \\ \frac{u_{l}(s) u_{r}(\rho)}{w}, & s<\rho,\end{cases}
$$

where $w:=J_{1}\left(\kappa r_{0}\right) N_{1}(\kappa r)-J_{1}(\kappa r) N_{1}\left(\kappa r_{0}\right), u_{l}(x):=J_{1}(\kappa x) N_{1}\left(\kappa r_{0}\right)-J_{1}\left(\kappa r_{0}\right)$ $\times N_{1}(\kappa x)$ and $u_{r}(x):=J_{1}(\kappa x) N_{1}(\kappa r)-J_{1}(\kappa r) N_{1}(\kappa x)$.

Using the same technique as in [20] we obtain an integral representation of solution $u(s)$ to equation $(3.4)$ for $s \in\left[r_{0}, r\right]$ :

$$
u(s)=-\alpha \int_{r_{0}}^{r} G(\rho, s) \rho u^{3}(\rho) d \rho+f(s),
$$

where $f(s)=-r u(r) G^{\prime}(r, s)=-C I_{1}(\beta r) u_{l}(s) w^{-1}$.

Assume that $f \in C\left[r_{0}, r\right]$. From the properties of the Green function, it follows that kernel $N(\rho, s ; \beta)$ is a continuous function in the square $r_{0} \leqslant \rho, s \leqslant$ $r$. From the results of [24] it follows that the nonlinear operator

$$
S(u):=\int_{r_{0}}^{r} N(\rho, s ; \beta) u^{3}(\rho) d \rho+f(s),
$$

where $N(\rho, s ; \beta):=-\alpha \rho G(\rho, s ; \beta)$, is completely continuous on each bounded subset of $C\left[r_{0}, r\right]$. Consider the cubic equation $\|\mathrm{N}\| x^{3}+\|f\|=x$, where

$$
\|\mathrm{N}\|=\max _{s \in\left[r_{0}, r\right]} \int_{r_{0}}^{r}|N(\rho, s)| d \rho \text { and }\|f\|=\max _{s \in\left[r_{0}, r\right]}|f(s)| .
$$

It can be shown (see [24]) that the following assertions are valid, which will be used below.

Proposition 1. Under the condition $0 \leqslant\|f\|<\frac{2}{3} \frac{1}{\sqrt{3\|\mathrm{~N}\|}}$, the equation $\|\mathrm{N}\| x^{3}+\|f\|=x$ has two nonnegative solutions

$$
\begin{aligned}
& x_{*}=-2 \sqrt{\frac{1}{3\|\mathrm{~N}\|}} \cos \left(\frac{1}{3} \arccos \left(\frac{3 \sqrt{3}}{2}\|f\| \sqrt{\|\mathrm{N}\|}\right)-\frac{2 \pi}{3}\right), \\
& x^{*}=-2 \sqrt{\frac{1}{3\|\mathrm{~N}\|}} \cos \left(\frac{1}{3} \arccos \left(\frac{3 \sqrt{3}}{2}\|f\| \sqrt{\|\mathrm{N}\|}\right)+\frac{2 \pi}{3}\right),
\end{aligned}
$$

such that $x_{*}<x^{*}$. 
Proposition 2. Under the condition $\|f\| \leqslant \frac{2}{3} \frac{1}{\sqrt{3\|\mathrm{~N}\|}}$, equation (4.2) has at least one solution such that $\|u\| \leqslant x^{*}$.

Using Propositions 1 and 2, it can be shown (see [24]) that the following theorems hold.

Theorem 1. If $\alpha \leqslant A^{2}$, where $A=2 /\left(3\|f\| \sqrt{3\left\|\mathrm{~N}_{1}\right\|}\right)$,

$\left\|\mathrm{N}_{1}\right\|=\max \int_{r_{0}}^{r}|\rho G(\rho, s)| d \rho$, then equation (4.2) has a unique continuous solution $u \in C\left[r_{0}, r\right]$ such that $\|u\| \leqslant x_{*}$.

Theorem 2. Let the kernel $N$ and the right-hand side $f$ of integral equation (4.2) be continuous functions of the parameter $\beta \in B_{0}, N(\rho, s ; \beta) \subset$ $C\left(\left[r_{0}, r\right] \times\left[r_{0}, r\right] \times B_{0}\right), f(s, \beta) \subset C\left(\left[r_{0}, r\right] \times B_{0}\right)$, on a certain interval $B_{0}$ of the real axis. Assume in addition that

$$
0<\|f(\beta)\|<2 /(3 \sqrt{3\|\mathrm{~N}(\beta)\|}) .
$$

Then solution $u(\rho ; \beta)$ to equation (4.2) for $\beta \in B_{0}$ exists, is unique, and depends continuously on parameter $\beta, u(\rho ; \beta) \in C\left(\left[r_{0}, r\right] \times B_{0}\right)$.

Taking the derivative with respect to $s$ of expression (4.2), setting $s=r$ in equation (4.2), using transmission conditions (3.7), and taking into account solutions (3.6), we obtain a characteristic equation for eigenvalues $\beta$ usually the called dispersion equation (DE)

$$
C g(\beta)=\alpha F(\beta),
$$

where

$$
\begin{aligned}
& F(\beta)=\kappa \frac{\pi}{2} \frac{J_{1}\left(\kappa r_{0}\right) N_{1}^{\prime}(\kappa r)-J_{1}^{\prime}(\kappa r) N_{1}\left(\kappa r_{0}\right)}{J_{1}\left(\kappa r_{0}\right) N_{1}(\kappa r)-J_{1}(\kappa r) N_{1}\left(\kappa r_{0}\right)} \int_{r_{0}}^{r} \rho u_{r}(\rho) u^{3}(\rho) d \rho, \\
& g(\beta)=\mu \beta I_{1}^{\prime}(\beta r)+\kappa I_{1}(\beta r) \frac{J_{1}\left(\kappa r_{0}\right) N_{1}^{\prime}(\kappa r)-J_{1}^{\prime}(\kappa r) N_{1}\left(\kappa r_{0}\right)}{J_{1}\left(\kappa r_{0}\right) N_{1}(\kappa r)-J_{1}(\kappa r) N_{1}\left(\kappa r_{0}\right)} .
\end{aligned}
$$

\section{Existence of solution of the dispersion equation}

Note first that zeros of the function $\Phi(\beta) \equiv C g(\beta)-\alpha F(\beta)$ are the values of $\beta$ for which problem $P$ has non-trivial solutions.

Let us analyze the existence of solutions of DE for the linear problem. From equation (4.3) with $\alpha=0$, we obtain

$$
\mu \beta \frac{I_{1}^{\prime}(\beta r)}{I_{1}(\beta r)}=\kappa \frac{J_{1}\left(\kappa r_{0}\right) N_{1}^{\prime}(\kappa r)-J_{1}^{\prime}(\kappa r) N_{1}\left(\kappa r_{0}\right)}{J_{1}\left(\kappa r_{0}\right) N_{1}(\kappa r)-J_{1}(\kappa r) N_{1}\left(\kappa r_{0}\right)} .
$$

The left-hand side of (5.1) is bounded, positive, and continuous for positive $\beta$ [1]. It is known [1] that the right-hand side of (5.1) has simple poles $\beta_{1}^{(n)}$, $\beta_{1}^{(n)}$ is the $n$th positive root of the equation

$$
J_{1}\left(\sqrt{\chi^{2}-\beta^{2}} r_{0}\right) N_{1}\left(\sqrt{\chi^{2}-\beta^{2}} r\right)-J_{1}\left(\sqrt{\chi^{2}-\beta^{2}} r\right) N_{1}\left(\sqrt{\chi^{2}-\beta^{2}} r_{0}\right)=0,
$$


$n=1,2, \ldots$ It follows from the properties of the Bessel functions that the right-hand side of (5.1) is a decreasing function.

We denote by $\beta_{1}^{\prime(n)}$ the $n$th positive root of the equation

$$
J_{1}\left(\sqrt{\chi^{2}-\beta^{2}} r_{0}\right) N_{1}^{\prime}\left(\sqrt{\chi^{2}-\beta^{2}} r\right)-J_{1}\left(\sqrt{\chi^{2}-\beta^{2}} r\right) N_{1}^{\prime}\left(\sqrt{\chi^{2}-\beta^{2}} r_{0}\right)=0,
$$

$n=1,2, \ldots$ This means that there is at least one root $\beta_{0}^{(n)}$ of Equation (5.1) in each interval $\left(\beta_{1}^{(n)}, \beta_{1}^{\prime(n)}\right), n=1,2, \ldots$

The following Lemma 1 give criteria for the solvability of the above-formulated linear problems:

Lemma 1. It is possible to choose $r>0$ in such a way that Equation (5.1) would have $n$ roots $\beta_{0}^{(1)}, \ldots, \beta_{0}^{(n)}$, such that the inequalities

$$
0<\beta_{0}^{(1)}<\ldots<\beta_{0}^{(n)}<\chi
$$

hold.

Now, we can show that solutions of the equation $\Phi(\beta)=0$ exists. Choose sufficiently small numbers $\delta_{i}>0$ such that the Green function exists and is continuous on the union $B:=\bigcup_{i=1}^{n} B_{i}$ of the intervals

$$
B_{i}:=\left[\beta_{1}^{(i)}+\delta_{i}, \beta_{1}^{(i)}\right], \quad i=\overline{1, n} .
$$

In addition, assume that $\delta_{i}$ are chosen so that the inequalities

$$
g\left(\beta_{1}^{(i)}+\delta_{i}\right) g\left(\beta_{1}^{(i)}\right)<0, \quad i=\overline{1, n}
$$

are valid.

In other words, we choose $\delta_{i}$ so that to move away from the poles of the Green function $\beta_{1}^{(n)}$ but preserve solutions of the $\operatorname{DE} g(\beta)=0$ for the linear medium. Thus, we can choose a sufficiently small value of $\alpha$ such that the nonlinear DE $C g(\beta)-\alpha F(\beta)=0$ will have a solution which lies close to the "linear" solution.

It should be pointed out that $F(\beta)$ is bounded and the product $\alpha F(\beta)$ can be made sufficiently small by choosing a suitable $\alpha_{0}$. Then, it can be affirmed that $C g(\beta)-\alpha F(\beta)=0$ has a solution if $\alpha_{0}$ is small enough.

Consider the $\operatorname{DE} \Phi(\beta)=0$. Function $g(\beta)$ is continuous and changes its sign when $\beta$ varies from $\beta_{1}^{\prime(i)}$ to $\beta_{1}^{(i)}+\delta_{i}$. Since $F(\beta)$ is bounded for $\beta$ varying from $\beta_{1}^{\prime(n)}$ to $\beta_{1}^{(n)}+\delta_{i}$, we can always assure, by choosing a suitable $\alpha$, that the equation $\Phi(\beta)=0$ has at least $n$ roots $\beta_{i}$ such that $\beta_{i} \in B_{i}, i=\overline{1, n}$.

The main result of the present work is the following theorem.

Theorem 3. Let $B_{i}, i=\overline{1, n}$ be a segment defined by formula (5.2). Then there is a number $\alpha_{0}>0$ such that, for any $\alpha \leqslant \alpha_{0}$, problem $P$ has at least $n$ eigenvalues $\beta_{i}$ such that $\beta_{i} \in B_{i}$. 
Proof. The Green function exists for all $\beta \in B$. Clearly,

$$
A(\beta)=2 /\left(3\|f(\beta)\| \sqrt{3\left\|\mathrm{~N}_{1}(\beta)\right\|}\right)
$$

is a continuous function of $\beta \in \Gamma$. Let $A_{1}=\min _{\beta \in \Gamma} A(\beta)$ and assume that $\alpha<A_{1}^{2}$. According to Theorems 1 and 2 , there is a unique solution $u=u(\beta)$ to equation (3.6) for any $\beta \in \Gamma$. This solution is a continuous function, and $\|u\| \leqslant x_{*}=x_{*}(\beta)$. Let $x_{00}=\max _{\beta \in \Gamma} x_{*}(\beta)$. Estimating $F(\beta)$, we obtain $|F(\beta)| \leqslant$ $C_{0} x_{00}^{3}$, where $C_{0}$ is a constant.

Function $g(\beta)$ is continuous, and the equation $g(\beta)=0$ has at least one root $\beta_{i}$ inside the interval $\Gamma_{i}, \beta_{1}^{(i)}+\delta_{i}<\beta_{i}<\beta_{1}^{(i)}$. Let $M_{1}=\min _{1 \leqslant i \leqslant n}\left|g\left(\beta_{1}^{(i)}+\delta_{i}\right)\right|$, $M_{2}=\min _{1 \leqslant i \leqslant n}\left|g\left(\beta_{1}^{\prime(i)}\right)\right|$. Then, $\widetilde{M}=\min \left\{M_{1}, M_{2}\right\}$ is positive and independent of $\alpha$.

If $\alpha \leqslant \frac{\widetilde{M}}{C_{0} x_{00}^{3}}$, then

$$
\left(C g\left(\beta_{1}^{(i)}+\delta_{i}\right)-\alpha F\left(\beta_{1}^{(i)}+\delta_{i}\right)\right) \times\left(C g\left(\beta_{1}^{(i)}\right)-\alpha F\left(\beta_{1}^{(i)}\right)\right)<0 .
$$

Function $C_{1} g(\beta)-\alpha F(\beta)$ is continuous; hence, the equation $C_{1} g(\beta)-$ $\alpha F(\beta)=0$ has a root $\beta_{i}$ inside $\Gamma_{i}$; that is, $\beta_{1}^{(i)}+\delta_{i}<\beta_{i}<\beta_{1}^{\prime(i)}$. Finally, we can choose $\alpha_{0}=\min \left\{A_{1}^{2}, \frac{\widetilde{M}}{C_{0} x_{00}^{3}}\right\}$ which completes the proof.

Theorem 3 implies that, under the above-formulated conditions, there exist leaky TE-polarized wave propagating without attenuation in a metamaterial Goubau Line filled with an isotropic Kerr-nonlinear medium.

\section{$6 \quad$ Numerical results}

Numerical results are obtained with the help of a version of the shooting method (see e.g. $[16,23]$ ). In the figures below, normalized eigenvalues $\beta / k_{0}$ are shown calculated with respect to frequency $\omega$, as well as eigenfunctions $u(\rho)$.

The following values of parameters are used for calculations in Figures 2-5: $\varepsilon=4, \mu=1, C_{1}=1, r_{0}=1$, and $r=3 \mathrm{~mm}$. Blue lines correspond to the linear case and red curves to the nonlinear case. The vertical dashed line corresponds to the frequency value $\omega=90 \mathrm{GHz}$. Eigenvalues of the problem are points of intersections of this dashed line with the dispersion curves: two points in Figures 2 and 4 are marked (the smallest value, a red dot, corresponds to the nonlinear case, while the blue dot to the linear case). The domain under grey dashed line is the domain where the linear problem has a solution.

In the figures for eigenfunctions the colour of a curve duplicates the colour of the marked eigenvalue on the dispersion curves. The larger an eigenvalue, the higher is the maximum of the corresponding eigenfunction. The vertical grey dashed line corresponds to the dielectric-free space boundaries.

If we compare blue (linear case) and red (nonlinear case) dispersion curves, we see that there are eigenvalues that are close to those occurring in the linear 


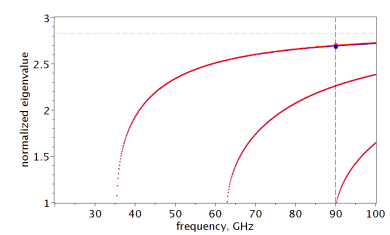

Figure 2. Nonlinearity coefficient $\alpha=2 \times 10^{-5} \mathrm{~mm} / \mathrm{V}$. Marked eigenvalues: linear eigenvalue $\beta \approx 5.0815$

(blue dot); nonlinear eigenvalue $\beta \approx 5.0893$ (red dot).

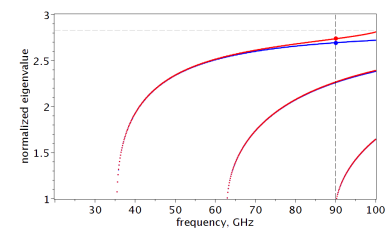

Figure 4. Nonlinearity coefficient $\alpha=2 \times 10^{-4} \mathrm{~mm} / \mathrm{V}$. Marked eigenvalues: linear eigenvalue $\beta \approx 5.0815$

(blue dot); nonlinear eigenvalue $\beta \approx 5.1623$ (red dot).

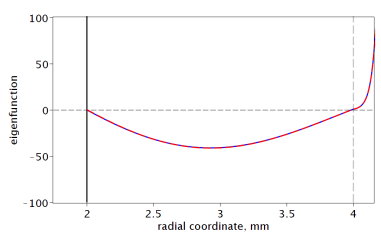

Figure 3. Nonlinearity coefficient $\alpha=2 \times 10^{-5} \mathrm{~mm} / \mathrm{V}$. Eigenfunctions.

Blue dash line (linear problem) $\beta \approx 5.0815$ and red line (nonlinear problem) $\beta \approx 5.0893$.

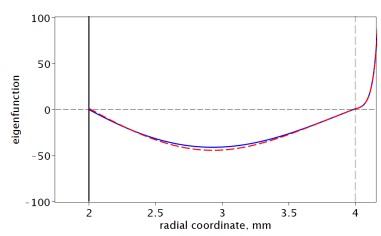

Figure 5. Nonlinearity coefficient $\alpha=2 \times 10^{-4} \mathrm{~mm} / \mathrm{V}$. Eigenfunctions.

Blue dash line (linear problem) $\beta \approx 5.0815$ and red line (nonlinear problem) $\beta \approx 5.1623$.

case; the latter can be determined with the help of perturbation theory and existence of these very eigenvalues is rigorously proved in this paper.

\section{Conclusions}

We have developed a method of analysis of the leaky TE wave propagation in a Goubau Line with a nonlinear metamaterial filling. The existence of these waves has been proved by reducing the problem to a nonlinear integral equation and applying the method of contraction. An analytical method proposed in this study allows one to prove the existence of eigenvalues of the nonlinear problem that are close to eigenvalues of the linear problem and are actually perturbations of the latter. Numerical solution is performed using a version of the shooting method developed specifically for this class of problems.

The proposed technique and mathematical method can be developed and applied to rigorous validation of the existence of other types of waves and analysis of metamaterial dielectric waveguides having different cross sections.

\section{Acknowledgements}

This work was supported by the Russian Science Foundation, project no. 2011-20087. 


\section{References}

[1] M. Abramowitz and I. Stegun. Handbook of Mathematical Functions with Formulas, Graphs, and Mathematical Tables. Dover Publications, 1965.

[2] M.J. Adams. An Introduction to Optical Waveguides. New York: Wiley, 1951.

[3] N.N. Akhmediev and A.Ankevich. Solitons, Nonlinear Pulses and Beams. Chapman and Hall, London, 1997.

[4] G. Goubau. Surface waves and their application to transmission lines. Journal of Applied Physics, 21(11):1119-1128, 1950. https://doi.org/10.1063/1.1699553.

[5] G. Goubau. Open wire lines. IRE Trans. Microwave Theory and Technique, 4(4):197-200, 1956. https://doi.org/10.1109/TMTT.1956.1125062.

[6] F. Harms. Elektromagnetische wellen an einem draht mit isolierender zylindrischer hülle. Annals of Physics, 328(6):44-60, 1907. https://doi.org/10.1002/andp.19073280603.

[7] L.D. Landau, E.M. Lifshitz and L.P. Pitaevskii. Course of Theoretical Physics. Electrodynamics of Continuous Media. Butterworth-Heinemann, Oxford, 1993.

[8] N. Marcuvitz. On field representations in terms of leaky modes or eigenmodes. IRE Transactions on Antennas and Propagation, 4(3):192-194, 1956. https://doi.org/10.1109/TAP.1956.1144410.

[9] F. Monticone and A. Alu. Leaky-wave theory, techniques, and applications: From microwaves to visible frequencies. In Proceedings of the IEEE, volume 103(5), pp. 793-821. IEEE, 2015. https://doi.org/10.1109/JPROC.2015.2399419.

[10] A.A. Oliner. Leaky waves: Basic properties and applications. In Proceedings of 1997 Asia-Pacific Microwave Conference, volume 1, pp. 397-400, Hong Kong/China, 1997. IEEE. https://doi.org/10.1109/APMC.1997.659407.

[11] V.P. Silin P.N. Eleonskii, L.G. Oganes'yants. Cylindrical nonlinear waveguides. Soviet Physics Jetp, 35:44-47, 1972.

[12] R.A. Shelby, D.R.Smith and S.Schultz. Experimental verification of a negative index of refraction. Science, 292(5514):77-79, 2001. https://doi.org/10.1126/science.1058847.

[13] Y.R. Shen. The Principles of Nonlinear Optics. John Wiley and Sons, New York, 1984.

[14] Yu. Smirnov and E. Smolkin. On the existence of non-polarized azimuthalsymmetric electromagnetic waves in circular dielectric waveguide filled with nonlinear isotropic homogeneous medium. Wave Motion, 77:77-90, 2018. https://doi.org/10.1016/j.wavemoti.2017.11.001.

[15] Yu. Smirnov, E. Smolkin and V. Kurseeva. The new type of non-polarized symmetric electromagnetic waves in planar nonlinear waveguide. Applicable Analysis, 98(3):483-498, 2019. https://doi.org/10.1080/00036811.2017.1395865.

[16] Yu. Smirnov, E. Smolkin and D. Valovik. Nonlinear double-layer bragg waveguide: Analytical and numerical approaches to investigate waveguiding problem. Advances in Numerical Analysis, 2014:1-11, 2014. https://doi.org/10.1155/2014/231498.

[17] E. Smolkin. Propagation of TE waves in a double-layer nonlinear inhomogeneous cylindrical waveguide. In Days on Diffraction'2014 Proceedings, pp. 204-209, Saint Petersburg, Russia, 2014. IEEE. https://doi.org/10.1109/DD.2014.7036451. 
[18] E. Smolkin. Goubau line filled with nonlinear medium: Numerical study of TMpolarized waves. In Proceedings of the 2015 17th International Conference on Electromagnetics in Advanced Applications, pp. 1572-1575, Turin, Italy, 2015. IEEE. https://doi.org/10.1109/ICEAA.2015.7297390.

[19] E. Smolkin. The azimuthal symmetric hybrid waves in nonlinear cylindrical waveguide. In Proceedings of the 2017 Progress in Electromagnetics Research Symposium, PIERS 2017, pp. 348-353, Saint Petersburg, Russia, 2017. IEEE. https://doi.org/10.1109/PIERS.2017.8261763.

[20] E. Smolkin and Yu. Shestopalov. Nonlinear Goubau line: analyticalnumerical approaches and new propagation regimes. Journal of Electromagnetic Waves and Applications, 31(8):781-797, 2017. https://doi.org/10.1080/09205071.2017.1317036.

[21] E. Smolkin, Yu. Shestopalov and M. Snegur. Diffraction of TM polarized electromagnetic waves by a nonlinear inhomogeneous metal-dielectric waveguide. In Proceedings of the 2017 19th International Conference on Electromagnetics in Advanced Applications, ICEAA 2017, pp. 1288-1291, VERONA ITALY, 2017. IEEE.

[22] E. Smolkin and D. Valovik. Numerical solution of the problem of propagation of TM-polarized electromagnetic waves in a nonlinear two-layered dielectric cylindrical waveguide. In Mathematical Methods in Electromagnetic Theory (MMET), 2012 International Conference on, pp. 68-71, Kharkiv, Ukraine, 2012. IEEE. https://doi.org/10.1109/MMET.2012.6331288.

[23] E. Smolkin and D. Valovik. Calculation of the propagation constants of inhomogeneous nonlinear double-layer circular cylindrical waveguide by means of the cauchy problem method. Journal of Communications Technology and Electronics, 58:762-769, 2013. https://doi.org/10.1134/S1064226913060132.

[24] E. Smolkin and D. Valovik. Guided electromagnetic waves propagating in a two-layer cylindrical dielectric waveguide with inhomogeneous nonlinear permittivity. Advances in Mathematical Physics, 2015, 2015. https://doi.org/10.1155/2015/614976.

[25] E. Smolkin and D. Valovik. Nonlinear propagation of coupled electromagnetic waves in a circular cylindrical waveguide. Computational Mathematics and Mathematical Physics, 57(8):1294-1309, 2017. https://doi.org/10.1134/S0965542517080127.

[26] A.W. Snyder and J. Love. Optical waveguide theory. Springer, 1983.

[27] A. Sommerfeld. Uber die fortpflanzung elektrodynamischer wellen längs eines drahtes. Annals of Physics, $\mathbf{3 0 3 ( 2 ) : 2 3 3 - 2 9 0 ,} 1899$. https://doi.org/10.1002/andp.18993030202.

[28] L.A. Vainstein. Electromagnetic Waves. Radio i svyaz, Moscow, 1988.

[29] V.G. Veselago. The electrodynamics of substances with simultaneously negative values of $\varepsilon$ and $\mu$. Soviet Physics Uspekhi, 10(4):509-514, 1968. https://doi.org/10.1070/PU1968v010n04ABEH003699.

[30] R.W. Ziolkowski and E.Heyman. Wave propagation in media having negative permittivity and permeability. PHYSICAL REVIEW E, 64(5), 2001. https://doi.org/10.1103/PhysRevE.64.056625. 